\title{
Piezoelectricity in PVDF and PVDF Based Piezoelectric Nanogenerator: A Concept
}

\author{
Binoy Bera ${ }^{1, *}$, Madhumita Das Sarkar ${ }^{2}$ \\ ${ }^{1,2}$ Department of computer science and engineering, West Bengal University of Technology, Kolkata - 700064, \\ India
}

\begin{abstract}
Polyvinylidene fluoride or simply PVDF is one of the most important semicrystalline polymers which generate piezoelectricity when a pressure or mechanical force applied on it. It has four crystalline phases $\alpha, \beta, \gamma$ and $\delta$ depending on the chain conformation structure. Among them $\alpha$ is non polar phase and $\beta$ and $y$ are polar phase. Piezoelectricity in PVDF arises due to the $\beta$ and $y$ phase formation. Several materials have been introduced for the preparation of nanogenerator. Among them PVDF (polyvinylidene fluoride) is most interesting material used in nanogenerator preparation due to its flexibility, bio- compatiable, nontoxic in nature. It is used in nanogenerator application due to its good ferroelectric, piezoelectric and pyroelectric properties. In this article we describe little bit concept about PVDF, its piezoelectricity and PVDF based nanogenerator. Application of piezoelectric nanogenerator is also briefly described here.
\end{abstract}

Keywords: PVDF, nanogenerator, piezoelectricity, $\beta$ phase, electrospinning, electroactive phase, Poling process.

\section{Introduction}

Polyvinylidene fluoride, or polyvinylidene difluoride, (PVDF) is a highly nonreactive thermoplastic fluoropolymer produced by the polymerization of vinylidene difluoride. PVDF has four crystalline phases $\alpha, \beta, \mathrm{y}$ and $\delta$ depending on the chain conformation. Among them $\alpha$ is thermodynamically most stable and non polar in nature. $\beta$ and $\gamma$ are polar phase. Non polar PVDF is used as insulator and painting. Polar PVDF is used in energy harvesting, sensor, actuator, electronics e.t.c. PVDF has several properties like biocompatiable, chemical resistance, good film forming capability, cost effictiveness e.t.c.. Among four crystalline phase $\beta$ is of great importance due to its spontaneous polarization and piezoelectric sensitivity. Because of this property tremendous effort has been paid to induce electroactive $\beta$ phase in PVDF. Several methods are there to induce $\beta$ phase in PVDF. It can be achieved by placing it into a high electric field which randomly orient $\mathrm{CH}_{2} / \mathrm{CF}_{2}$ dipoles along with the electrical field direction. Different types of doping (like metal nanoparticle, hydrated salt for avoiding poling step) also done for achieving electroactive phase. The dielectric constant of PVDF is also high about 12, which makes it suitable to integrate in device for getting singnal to noise ratio less. It has glass transition temperature of $-35^{\circ} \mathrm{C}$ which is much less than room temperature. So it is much flexible in room temperature. Now a days PVDF is largely used in nanogenerator application due to its piezoelectricity. PVDF is non toxic, flexible, easy to process make it suitable in nanogenerator application. PVDF or polyvinylidene fluoride is a polymer material which shows piezoelectricity when pressure or mechanical force applied on it. The material which shows piezoelectricity is called piezoelectric material. Piezoelectric material can be of crystals, ceramics or polymer. Piezoelectric crystals are like quartz, berilinite, gallium orthophosphate, and tourmaline.

Piezoelectric ceramics are like barium titanate, lead zirconate titanate, barium zirconate titanate. Some polymer (PVDF, PVDF - TrFE) also shows piezoelectric effect. Based on this properties a new type technology arises today. This is called nanogenerator. A nanogenerator is a type of device which generates voltage or power when a mechanical force or vibration applied on it. Among all piezoelectric material, polymer like PVDF is most suitable because it is nontoxic, flexible, bio- compatiable, high piezoelectric voltage constant. As already told PVDF exists in several forms: alpha (TGTG'), beta (TTTT), gamma (TTTGTTTG') and $\delta$ phases, depending on the chain conformations as trans (T) or gauche (G) linkages. Among them $\alpha$ does not show piezoelectric properties. $\beta, \gamma$ and $\delta$ phase show piezoelectric properties. Generally PVDF is present in non polar $(\alpha)$ phase. To make PVDF polar, several approaches are there like stretching, metal nanoparticle doping, hydrated salt dpoing, electrospinning process e.t.c. Among all polar phase $\beta$ phase is most important due to its high remnant polarization and highest dipolar moment per unit cell $(8 \times 10-30 \mathrm{C} \mathrm{m})$ when compared to the other two phases. FTIR, bands and X-ray diffraction peaks typically used for the identification of the phases. Many researchers are paying attention to nanogenerators (NGs) as energy source in self-powered mirco-nano system. Now a days device are gradually decreasing in size and they need small power. In that case nanogenerator is useful as it generates voltage in the range of micro and nano order. It is also used in small battery charging, wireless power transmission, LED light glow e.t.c. 


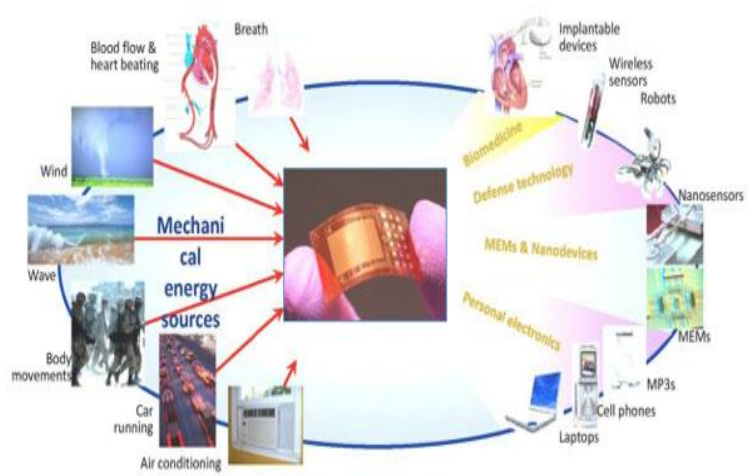

Fig.1. Different application of nanogenerator

\section{PVDF}

Polyvinylidene fluoride, or polyvinylidene difluoride, (PVDF) is a highly nonreactive thermoplastic fluoropolymer produced by the polymerization of vinylidene difluoride. As already told it has four crystalline phase depending on chain conformation structure. When poled, PVDF is a ferroelectric polymer, exhibiting efficient piezoelectric and pyroelectric properties. These characteristics make it useful in sensor and battery applications. PVDF has a glass transition temperature $\left(\mathrm{T}_{\mathrm{g}}\right)$ of about $-35^{\circ} \mathrm{C}$ and is typically 50-60\% crystalline. Unlike other popular piezoelectric materials, such as PZT (lead zirconate titanate), PVDF has a negative $d_{33}$ value. Physically, this means that PVDF will compress instead of expand or vice versa when exposed to the same electric field. PVDF is a specialty plastic used in applications requiring the highest purity, as well as resistance to solvents, acids and bases. Compared to other fluoropolymers, like polytetrafluoroethylene (Teflon), PVDF has a low density $\left(1.78 \mathrm{~g} / \mathrm{cm}^{3}\right)$. It is available as piping products, sheet, tubing, films, plate and an insulator for premium wire. It can be injected, molded or welded and is commonly used in the chemical, semiconductor, medical and defense industries, as well as in lithium-ion batteries. It is also available as a crosslinked closed-cell foam, used increasingly in aviation and aerospace applications.

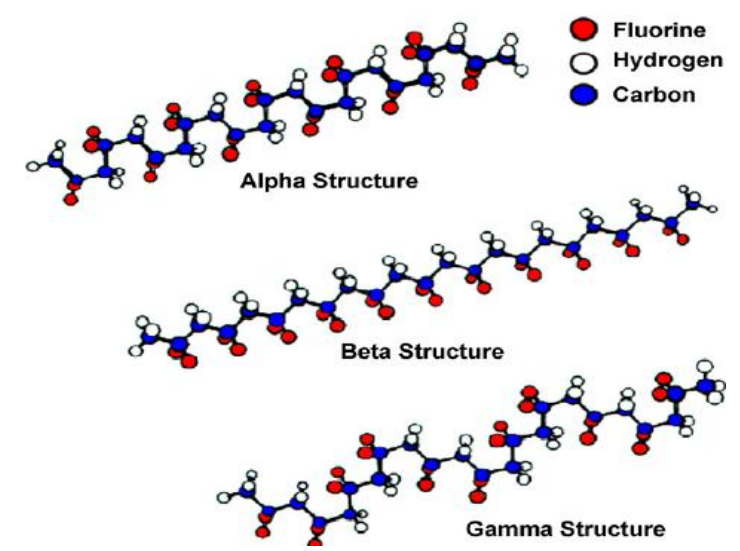

Fig.2. The three primary polymorphic crystalline phases of PVDF

\section{How Piezoelectricity Generates}

Piezoelectricity is the ability of certain material to generate electrical voltage when a mechanical force or pressure applied on it. Material which shows this kind of behavior is called piezoelectric material. PVDF is also a piezoelectric polymer material. As already mentioned, $\alpha$ phase of PVDF is non polar, so it does not show piezoelectric effect. $\beta$ and $y$ phase are responsible for generating piezoelectricity in it. Normally the charges of piezoelectric crystals are in balanced even they are not symmetrically arranged. The effects of charges are exactly cancel out, leaving no net charges in it. When a pressure or mechanical force applied on it, the charges are become out of balance. This effect of charges does not cancel each other, so net charges appears on both side of crystals. Such this way a voltage can be generate in it by simply pressing it. Now the piezoelectricity generation are depend on electroactive pahse and crystallinity of PVDF material. Normally PVDF material is not piezoelectric because it is in non polar phase. It is produced in large thin clear sheets which are then stretched and poled to give it the piezoelectric properties. Materials that have crystalline lattice structure, that is 
a three dimensional geometric arrangement of atoms in a molecule which repeats itself from molecule to molecule (unit cell). Piezoceramics are solid mixtures of minute piezo crystallites. Dipoles are randomly oriented initially. Repsonses of these dipoles to externally applied electric field would tend to cancel one another, producing no gross change in the dimensions of the piezoceramics. They do not have piezoelectric behavior at macro level. In order to obtain a useful macroscopic piezoelectric response, dipoles must be permanently aligned with one another. A process called poling is used for this purpose. A piezoelectric material has a characteristic Curie temperature. When heated above this temperature, dipoles can change oriention in the solid phase of the material. In poling process, material is heated above its Curie temperature and a strong electric field is applied. Direction of the electric field is the polarization direction and the dipoles shift into alignment with it. Keeping the electric field constant, material is cooled below its Curie temperature, with the results that the alignment of dipoles is permanently fixed. In this case, material is said to be poled.

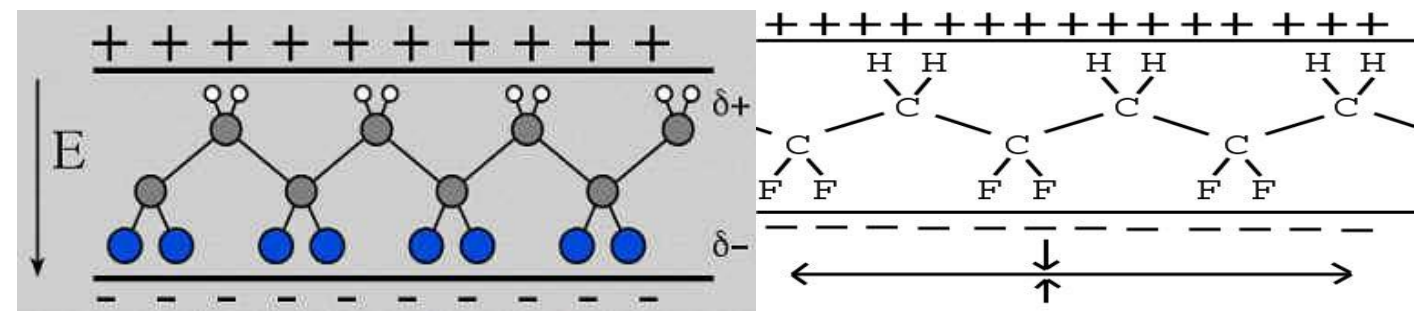

Fig.3. The electric field is in the opposite direction of the poled direction and the sheet is stretched length.
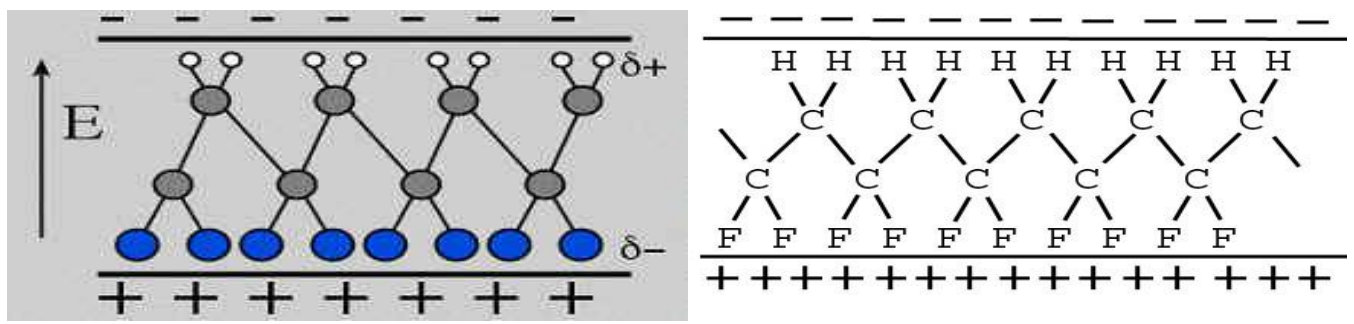

Fig.4. the electric field is in the same direction of the poled direction and the sheet is contracted in length.

\section{Nanogenerator}

A Nanogenerator is a type of technology that converts mechanical/thermal energy as produced by small-scale physical change into electricity. It has three typical approaches: piezoelectric, triboelectric, and pyroelectric nanogenerators. Both the piezoelectric and triboelectric nanogenerators can convert mechanical energy into electricity. However, pyroelectric nanogenerators can be used to harvest thermal energy from a time-dependent temperature fluctuation. Different materials like zinc oxide nanowire, lead zirconate titanate, barium titanate nanowire, PVDF e.t.c. are used for nanogenerator preparation. Nanogenerator is expected to be applied for various applications where the periodic kinetic energy exists, such as wind and ocean waves in a large scale to the muscle movement by the beat of a heart or inhalation of lung in a small scale. It is generally used in Self-powered nano/micro devices, Smart Wearable Systems, Transparent and Flexible Devices, Implantable Telemetric Energy Receiver e.t.c..
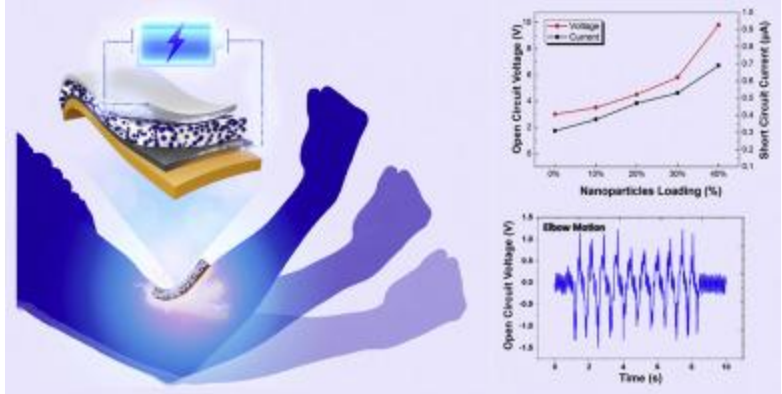

Fig.5. Nanogenerator gives output current from hand movement

\section{PVDF Based Nanogenerator And Application}

Now a days PVDF is used very widely in nanogenerator preparation. Ceramics have higher piezoelectric coefficient but their stiffness make them unsuitable under mechanical vibration. PVDF has great flexibility and good response in stress. Output voltage is dependent on the electroactive phase and crystallinity 
of PVDF. Due to specific chain conformation in crystal unit cell and providing highest remnant polarization, $\beta$ phase has attracted more attention than the others ( $\mathrm{\gamma}$ and $\delta$ ). A variety of methods have been developed to obtain such electroactive $\beta$-phase. Beta phase can be generated directly from electrospinning process. It generates fiber like structure and for nanogenerator preparation anode and cathode is placed on the both side of this fiber.

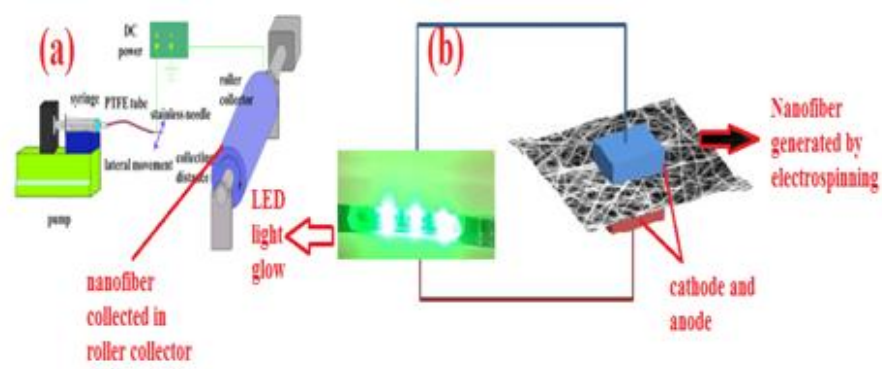

Fig.6. (a) PVDF nanofiber generated by electrospinning process. (b) nanogenerator as voltage source.

Output voltage can be generated from this nanogenerator by applying mechanical force or vibration on it. It can be placed in our human body and it generates voltage from human body motion due to its flexibility. Due to its biocompatiable nature, it can be placed inside our human body and from inside human body motion it generates voltage. PVDF based nanogenerator is used in different application like LED light glow, capacitor charging, wireless transmission.

\section{Conclusion}

PVDF generates voltage when pressure or mechanical force applied on it. The reverse process is also true that is when voltage is applied, it will vibrate. First property is applied in sensor application and reverse process is applied in actuator. For its biocompatiable property, it is used in biomedical sciences, like immunoblotting as an artificial membrane. PVDF membrane is also used in filtering devices. Piezoelectricity output depends on the percentage of electroactive phase in it. More electroactive phase generates more output voltage. Different materiasl are used to doped with PVDF for getting more piezoelectric voltage.

\section{Acknowledgement}

Binoy Bera wants to thank Dr. Madhumita Das Sarkar for her inspiration and guidance. Binoy also want to thank Dr. Dipankar Mandal of Jadavpur University for giving him a basic practical idea about PVDF and its piezoelectricity. Binoy Bera wants to thank TEQIPphaseII, MAKAUT (Refno:09.02/Regis/Appnt.4/5) for providing him the fellowship.

\section{References}

[1]. Zhang, Q. M., Bharti, V., Kavarnos, G., Schwartz, M. (Ed.), (2002). "Poly (Vinylidene Fluoride) (PVDF) and its Copolymers", Encyclopedia of Smart Materials, Volumes 1-2, John Wiley \& Sons, 807-825.

[2]. Kawai, Heiji (1969). "The Piezoelectricity of Poly (vinylidene Fluoride)". Japanese Journal of Applied Physics. 8 (7): 975.

[3]. Lolla, Dinesh; Gorse, Joseph; Kisielowski, Christian; Miao, Jiayuan; Taylor, Philip L.; Chase, George G.; Reneker, Darrell H (2015). "Polyvinylidene fluoride molecules in nanofibers, imaged at atomic scale by aberration corrected electron microscopy". Nanoscale.

[4]. Prevedouros K, Cousins IT, Buck RC, Korzeniowski SH (January 2006). "Sources, fate and transport of perfluorocarboxylates". Environ Sci Technol. 40 (1): 32-44.

[5]. Zhang, Qiwu; Lu, Jinfeng; Saito, Fumio; Baron, Michel (2001). "Mechanochemical solid-phase reaction between polyvinylidene fluoride and sodium hydroxide". Journal of Applied Polymer Science. 81 (9): 2249.

[6]. Guzman, E.; Cugnoni, J; Gmür, T (2013). "Survivability of integrated PVDF film sensors to accelerated ageing conditions in aeronautical/aerospace structures". Smart Mater Struct. 22 (6): 065020.

[7]. Omote, Kenji; Ohigashi, Hiroji; Koga, Keiko (1997). "Temperature dependence of elastic, dielectric, and piezoelectric properties of "single crystalline films of vinylidene fluoride trifluoroethylene copolymer". Journal of Applied Physics. 81 (6): 2760.

[8]. Liebert TC, Chartoff RP, Cosgrove SL, McCuskey RS. Subcutaneous implants of polypropylene filaments. J Biomed Mat Res 1976; 10: 939-51.

[9]. Laroche G, Marois Y, Schwarz E, Guidoin R, King MW, Paris E, Douville Y. Polyvinylidene fluoride monofilament sutures: can they be used safely for long-term anastomoses in the thoracic aorta? Artif Organs 1995; 19:1190-9.

[10]. Mary C, Marois Y, King M, Laroche G, Douville Y, Martin L, Guidoin R. Comparison of the in-vivo behaviour of polyvinylidenfluoride and polypropylene sutures in vascular surgery. ASAIO Journal 1998; 44:199-206.

[11]. Klinge U, Klosterhalfen B, Conze J, Limberg W, Obolenski B, Ottinger AP, Schumpelick V. Modified mesh for hernia repair that is adapted to the physiology of the abdominal wall. Eur J Surg 1998; 164:951-960.

[12]. Vinard E, Eloy R, Descotes J, Brudon J, Guidicelli H, Magne J, Patra P, Berruet R, Hue A, Chauchard J. Stability of performances of vascular prostheses retrospective study of 22 cases of human implanted prostheses. J Biomed Mat Res 1988; 22:633-648 
[13]. Maarek J, Guidon R, Aubin M, Prud'homme R. Molecular weight characterization of virgin and explanted polyester arterial prosthesis. J Biomed Mat Res 1984; 18:881-894.

[14]. B. Bera, Literature Review on Electrospinning Process (A Fascinating Fiber Fabrication Technique), Imperial Journal of Interdisciplinary Research (IJIR) Vol-2, Issue-8, 2016.

[15]. Berger K., Sauvage L. Late fiber deterioration in Dacron arterial grafts. Ann Surg 1980; 193: 477-491.

[16]. Riepe G, Loos J, Imig H, Schroder A, Schneider E, Petermann J, Rogge A, Ludwig M, Schenke A, Nassutt R, Chakfe N, Morlock M. Long-term in vivo alterations of polyester vascular grafts in humans. Eur J Vase Endovasc Surg 1997; 13:540-8.

[17]. Hauptmann S, Klosterhalfen B, Mittermayer C, RÜhlmann K, Kaufmann R, HÖcker H. Evidence for macrophage-mediated defluorization of a Teflon vascular graft. J Mat Sei: Mat Med 1996; 7: 345-348.

[18]. D Mandal, S Yoon, KJ Kim, Origin of Piezoelectricity in an Electrospun Poly (vinylidene fluoride-trifluoroethylene) Nanofiber Web-Based Nanogenerator and Nano-Pressure Sensor, Macromolecular rapid communications 32 (11), 831-837.

[19]. D Mandal, K Henkel, D Schmeißer, The electroactive $\beta$-phase formation in Poly (vinylidene fluoride) by gold nanoparticles doping, Materials Letters 73, 123-125.

[20]. SK Ghosh, TK Sinha, B Mahanty, D Mandal, Self-poled Efficient Flexible "Ferroelectretic" Nanogenerator: A New Class of Piezoelectric Energy Harvester, Energy Technology 3 (12), 1190-1197.

[21]. C. Chang, V. H. Tran, J. Wang, Y. K. Fuh, L. Lin, Direct-write piezoelectric polymeric nanogenerator with high energy conversion efficiency, 2010 Nano Lett. 10 726-31.

[22]. P. Juan, X. J. Yan, Y. D. Jiang, C. Chang and L. W. Lin, "Piezoelectric actuation of direct-write electrospun fibers," Sensor. Actuat. A- Phys., vol.164, pp. 131-136, 2010.

[23]. S. Bai, Q. Xu, L. Gu, F. Ma, Y. Qin, Z. L. Wang, single crystalline Lead Zirconate Titanae Nano/micro-wire based self-powered UV sensor, 2012 Nano Energy 1 789-95.

[24]. Lolla, Dinesh; Gorse, Joseph; Kisielowski, Christian; Miao, Jiayuan; Taylor, Philip L.; Chase, George G.; Reneker, Darrell H. (2015). "Polyvinylidene fluoride molecules in nanofibers, imaged at atomic scale by aberration corrected electron microscopy". Nanoscale.

[25]. Zhang, Qiwu; Lu, Jinfeng; Saito, Fumio; Baron, Michel (2001). "Mechanochemical solid-phase reaction between polyvinylidene fluoride and sodium hydroxide". Journal of Applied Polymer Science. 81 (9): 2249.

[26]. Omote, Kenji; Ohigashi, Hiroji; Koga, Keiko (1997). "Temperature dependence of elastic, dielectric, and piezoelectric properties of "single crystalline films of vinylidene fluoride trifluoroethylene copolymer". Journal of Applied Physics. 81 (6).

[27]. Bera, Binoy; Das Sarkar, Madhumita. "PVDF based Piezoelectric Nanogenerator as a new kind of device for generating power from renewable resources" . IOSR Journal of Polymer and Textile Engineering (IOSR-JPTE). Volume 4, Issue 2 (Mar. - Apr. 2017), PP 01-05. 